\title{
Microfabricated devices for performing chemical and biochemical analysis
}

$$
\because=
$$

J. M. Ramsey, S. C. Jacobson. and R. S. Foote

Oak Ridge National Laboratory

P.O. Box 2008

Oak Ridge, TN 37831.6142

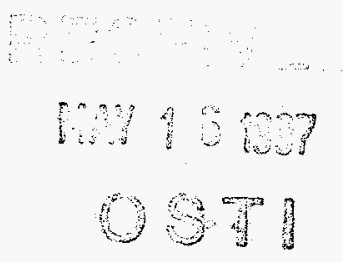

\section{Introduction}

There is growing interest in microfabricated devices that perform chemical and biochemical analysis. The general goal is to use microfabrication tools to construct miniature devises that can perform a complete analysis starting with an unprocessed sample. Such devices have been referred to as lab-on-a-chip devices. Initial efforts on microfluidic laboratory-on-a-chip devices focused on chemical separations. Several laboratories have reported over the past few years on devices microfabricated using planar glass substrates for performing capillary electrophoresis [1.2,3.4.5.6,7,8,9]. Microchip devices have been demonstrated that perform open channel electrochromatography [10], and micellar electrokinetic capillary chromatography [11]. Micromachined glass substrates have also been used for the separation of DNA fragments $[12,13]$. These miniature devices have shown performance either equivalent to or better than conventional laboratory devices in all cases investigated. For example, injection performance with the microfabricated devices has been observed to be one to two orders of magnitude more reproducible than with conventional capillary electrophoresis with $100 \mathrm{pL}$ volumes. Other miniature chemical analysis devices that have recently been reported include flow injection analysis [14, 15, 16] and biosensors [17].

More recently, monolithically integrated devices that embrace the concept of the lab-on-a-chip have been demonstrated. These devices include chemical reactions and separations micromachined into a single structure with reactions occurring prior to separation [18], [19] and following separation [20]. One of these integrated devices performs an enzymatic digestion of DNA and electrophoretic sizing of the fragments [19].

The microfabricated devices that we have been working with have demonstrated the ability to manipulate very small volumes of fluid ( $=$

"The submited manuscrip! nas deen authored by a contractor of the U.S. authored by a contractor of the U.S. Govemment under contract No. DE-
ACO5-96OR22464. Accordingly, the U.S. Government retains a nonexclusive, royalty-free license to

publish or reproduce the published

form of this contribution, or allow

others to do so, for U.S. Government purposes."

Research sponsored by Office of Research and Development, U.S. Department of Energy, under Contract DE-AC05-960R22464 with Oak Ridge National Laboratory, managed by Lockheed Martin Energy Research Corporation. 


\section{DISCLAIMER}

This report was prepared as an account of work sponsored by an agency of the United States Government. Neither the United States Government nor any agency thereof, nor any of their employees, make any warranty, express or implied, or assumes any legal liability or responsibility for the accuracy, completeness, or usefulness of any information, apparatus, product, or process disclosed, or represents that its use would not infringe privately owned rights. Reference herein to any specific commercial product, process, or service by trade name, trademark, manufacturer, or otherwise does not necessarily constitute or imply its endorsement, recommendation, or favoring by the United States Government or any agency thereof. The views and opinions of authors expressed herein do not necessarily state or reflect those of the United States Government or any agency thereof. 


\section{DISCLAMMER}

Portions of this document may be illegible in electronic image products. Images are produced from the best available original document. 
$100 \mathrm{pL}$ ) with high precision (< $1 \%$ rsd). The ability to combine reagents and perform chemical reactions "on-chip" suggests the eventual ability to perform virtually any type of "wet-chemical" bench procedure on a microfabricated device. The paradigm shift of moving the laboratory to a chip includes the advantages of reducing reagent volumes by four to six orders of magnitude, automated manipulations with no moving parts, reduced costs, highly parallel chemical processing, and higher processing speed. The volume of fluids that are manipulated or dispensed in the microfluidic structures discussed above is on the nanoliter scale or smaller versus tens of microliters at best for the conventional laboratory, corresponding to a reduction of $10^{4}$ or more! Flow rates on the devices that we have been studying are of the order of $1 \mathrm{~mL} / \mathrm{yr}$ of continuous operation implying the ability to incorporate reagents "on-board" the chip. By implementing multiple processes in a single device (integration), these small fluid quantities can be manipulated from process to process efficiently and automatically under computer control.

There are many potential applications of these fluidic microchip devices. Some applications such as chemical process control or environmental monitoring would require thit a chip be used over an extended period of time or for many analyses. Other applications such as forensics, clinical diagnostics, and genetic diagnostics would employ the chip devices as single use disposable devices. Additional potential applications such as genome sequencing and drug discovery include laboratory settings where high through-put analyses are desired. In analogy with the microelectronics paradigm, the later devices would attempt massively parallel chemical analysis.

\section{Microfabrication and microfluidics}

Our laboratory is currently involved in a program to investigate the possibility of microfabricating miniature instruments $\mathrm{that}$ perform conventional chemical analysis procedures. Specifically, we are investigating microdevices for performing the analysis of liquid borne materials. The approach Figure 2,1: Serpentine microchip. taken is to micromachine

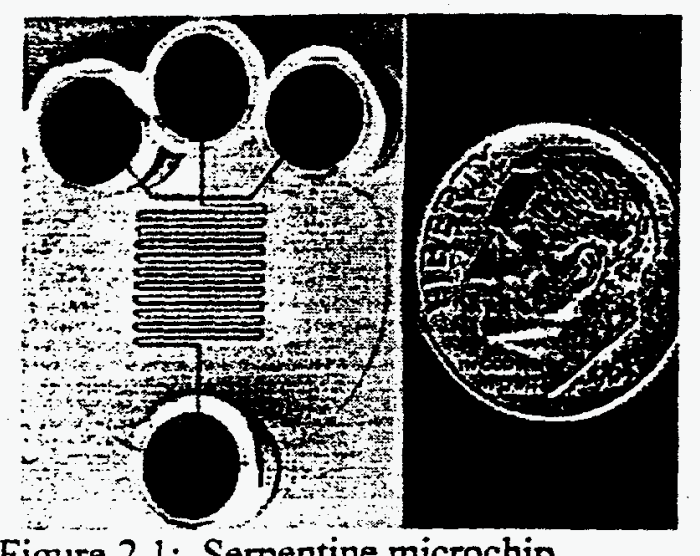


strength, and $h$ is the viscosity of the solvent. For reference, room temperature water in a glass tube moves at a velocity of $1 \mathrm{~cm} / \mathrm{s}$ with a field strength of $1500 \mathrm{~V} / \mathrm{cm}[21]$.

There are several advantages to using electroosmotic flow (EO) for microfluidic manipdfatons. The flow profile obtained with EO flow is planar. That is, the velocity is uniform everywhere within the cross section of the tube up to the double layer which has a typical thickness measured in tens of nanometers. Poiseuille flow, generated by hydrostatic pressure produces a parabolic flow profile, i.e. the velocity varies continuously with radius and is lowest at the walls and highest at the tube center. EO flow does not disperse material of a given type along the tube axis as the transverse velocity gradient in Poiseuille flow does. The EO flow profile allows for more efficient material transport because of minimal axial dispersion and provides a uniform flux of material throughout the channel cross section, i.e., there are no stagnation regions. Finally, fluid flows can be manipulated (valved) with no mechanical parts as described below.

Charged species also have a velocity component due to electrophoretic movement in an electric field. The velocity of a charged molecule under an electric field is the sum $\mathrm{Oi}^{\circ}$ the electrophoretic mobility, $\mu_{\text {ep }}$, and the electroosmotic mobility, i.e., the velocity of the solvent, as shown in equation 2 .

2)

$$
v_{\text {ion }}=\left(\mu_{e p}+\mu_{e o}\right) E
$$

Equation 2 implies that reagents will move with different velocities under the influence of an electric field and thus will arrive at a reaction site at different times. It is important to point out that the relative concentrations of the reagents will be identical to that in the reservoir from which they are pumped once the slowest moving component arrives at a particular site.

We have developed two different types of valves for microchip separations. One scheme is called a "constant volume valve" which is schematically described in Figure 2.2. The channels are connected to the separate reservoirs for sample.

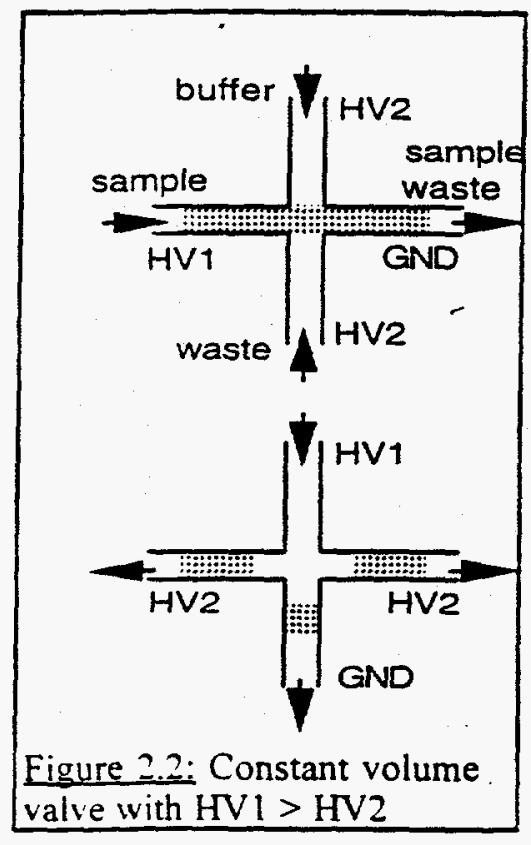


buffer, sample waste and waste as indicated. The potential distribution is initially configured as shown in the top half of Figure 2.2, where sample ions are pumped electrokinetically from the sample reservoir to the sample waste reservoir. Potentials are also placed on the buffer and waste reservoirs to provide a small amount of flow from the respective channels into the channel intersection. This additional flow spatially confines the sample to roughly the physical dimension of the channel intersection and is stable for constant potentials. The plug of fluid to be injected is representative of the sample in the reservoir after the slowest migrating ion arrives at the intersection. The potentials are then redistributed as shown in the lower half of Figure 2.2 to push the sample at the intersection into the separation channel. We have been able to inject $\approx 100 \mathrm{pL}$ volumes with a run-to-run reproducibility of $=0.3 \%$ rsd for peak areas using this technique. This reproducibility is one to two orders of magnitude better than conventional methods.

A second method was developed to inject volumes of arbitrary size and to provide unidirectional flow in the separation channel. This approach is called a "variable volume valve" or "gated valve" which is schematically shown in Figure 2.3. The same solution reservoirs are utilized but the positions of the sample and buffer reservoirs are exchanged. The potential distribution is now set as indicated in Figure 2.3 so that a strong flow of buffer goes to both waste reservoirs. The buffer flow provides fresh buffer to the separation channel and also pushes the sample stream to

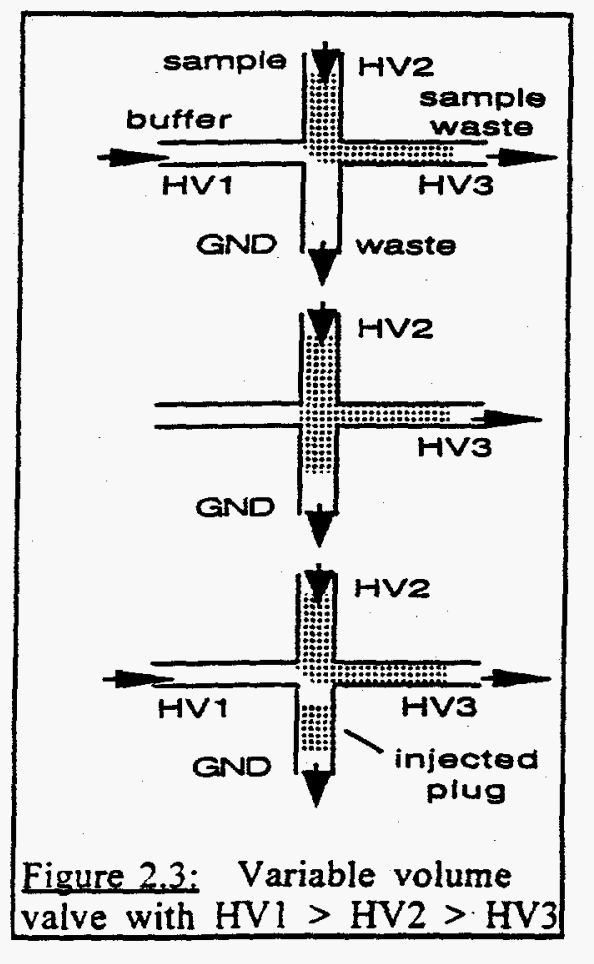
the sample waste reservoir.

To make an injection the potential is removed from the buffer reservoir so that there is no potential drop between the intersection and this reservoir. Sample now flows into the separation channel similar to an electrokinetic injection for as long as the potential is removed. Upon reapplication of the buffer reservoir potential, the sample stream is again pushed to waste. This injection scheme can provide larger injection volumes but has an electrophoretic mobility 
based bias. The gated injection is also quite reproducible with results for one specific implementation of $<1.8 \%$ rsd for injection volumes greater than $\approx 250 \mathrm{pL}$. We have also used this type of injection to perform "on-chip" sample stacking [9] by placing the sample in a lower conductivity buffer. Both of these injection techniques have been performed with and without electroosmotic flow.

\section{Chemical Separations}

As mentioned above, the initial demonstrations of such microfluidic devices involved chemical separations such as capillary electrophoresis and liquid chromatography. Up to 150,000 plates have been generated in less than one minute using micromachined separation devices. The serpentine device shown above in Fig. 2.1 has generated greater than 40,000 plates for electrophoretic separations using modest separation field strengths, e.g. 50 to $300 \mathrm{~V} / \mathrm{cm}$. The band broadening due to the serpentine geometry has been studied and is dependent primarily on the channel width. Channel widths of $\approx 30 \mu \mathrm{m}$ or less produce minimal band broadening effects [6].

Speed advantages can also accrue from miniaturization of chemical instruments as in microelectronics. Because the small dimensions permit lower currents and efficient heat dissipation, h i g her separation f i e 1 d strengths can be employed which result in higher efficiencies and shorter a $n$ a $l$ y $s$ is times. Speed advant ages also result from the

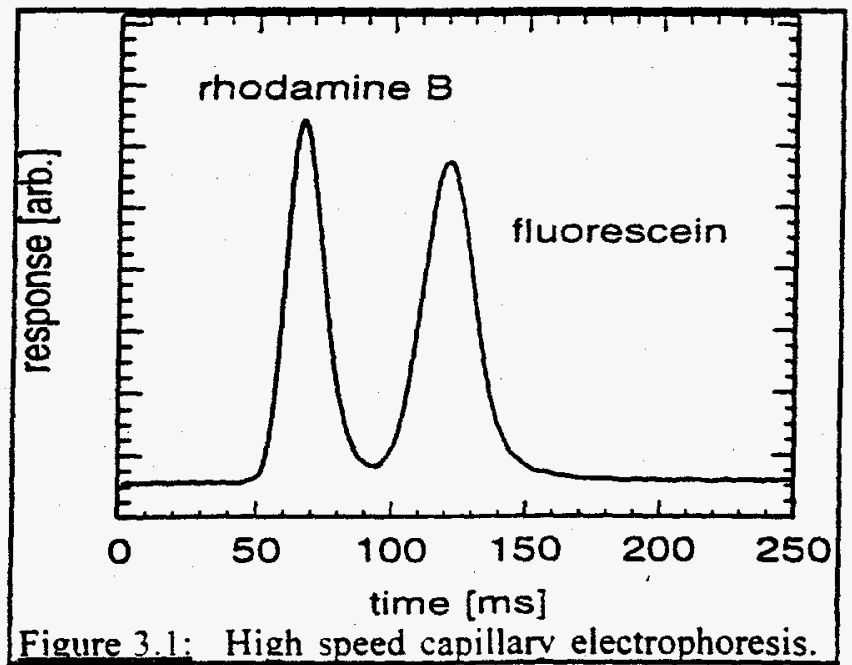
short injection plugs that can be generated with the micromachined devices. In Figure 3.1, for a separation length of $0.9 \mathrm{~mm}$, electrophoretic separations with baseline resolution are achieved in less than $150 \mathrm{~ms}$ with an electric field strength of $1.5 \mathrm{kV} / \mathrm{cm}$ and an efficiency of 1820 plates per second (Figure 3.1). For a separation length of $11.1 \mathrm{~mm}$, a minimum plate height of $0.7 \mu \mathrm{m}$ and a maximum 
number of plates per second of 18600 were achieved. These numbers are the highest number of theoretical plates per second reported. The latter figure of merit indicates that it is theoretically possible to analyze $\approx 50$ compounds per second.

We have also studied the separation of neutral species by open channel electrochromatography $[10]$ and micellar electrokinetic capillary chromatography (MECC) [11]. These two schemes employ electroosmotic flow to pump the samples through the channel manifold. For the electrochromatography, the surface of the channel wall was chemically modified with octadecylsilane to function as the stationary phase. In Figure 3.2, three organic dyes, coumarin 440 (C.440), coumarin 450 (C450), and coumarin 460 (C460), are resolved. Electroosmotic flow was used to "load" the sample into the microchip and to "pump" the mobile phase during the experiments. For electric field strengths of 27 to 163 $\mathrm{V} / \mathrm{cm}$, the linear velocity for the electroosmotic flow ranged from 0.13 to $0.78 \mathrm{~mm} / \mathrm{s}$. Detection was performed using direct fluorescence for separation monitoring a n d indirect fluorescence for void time measurements. Plate heights as low as $4.1 \mu \mathrm{m}$ and $5.0 \mu \mathrm{m}$ were generated for unretained and retained components,
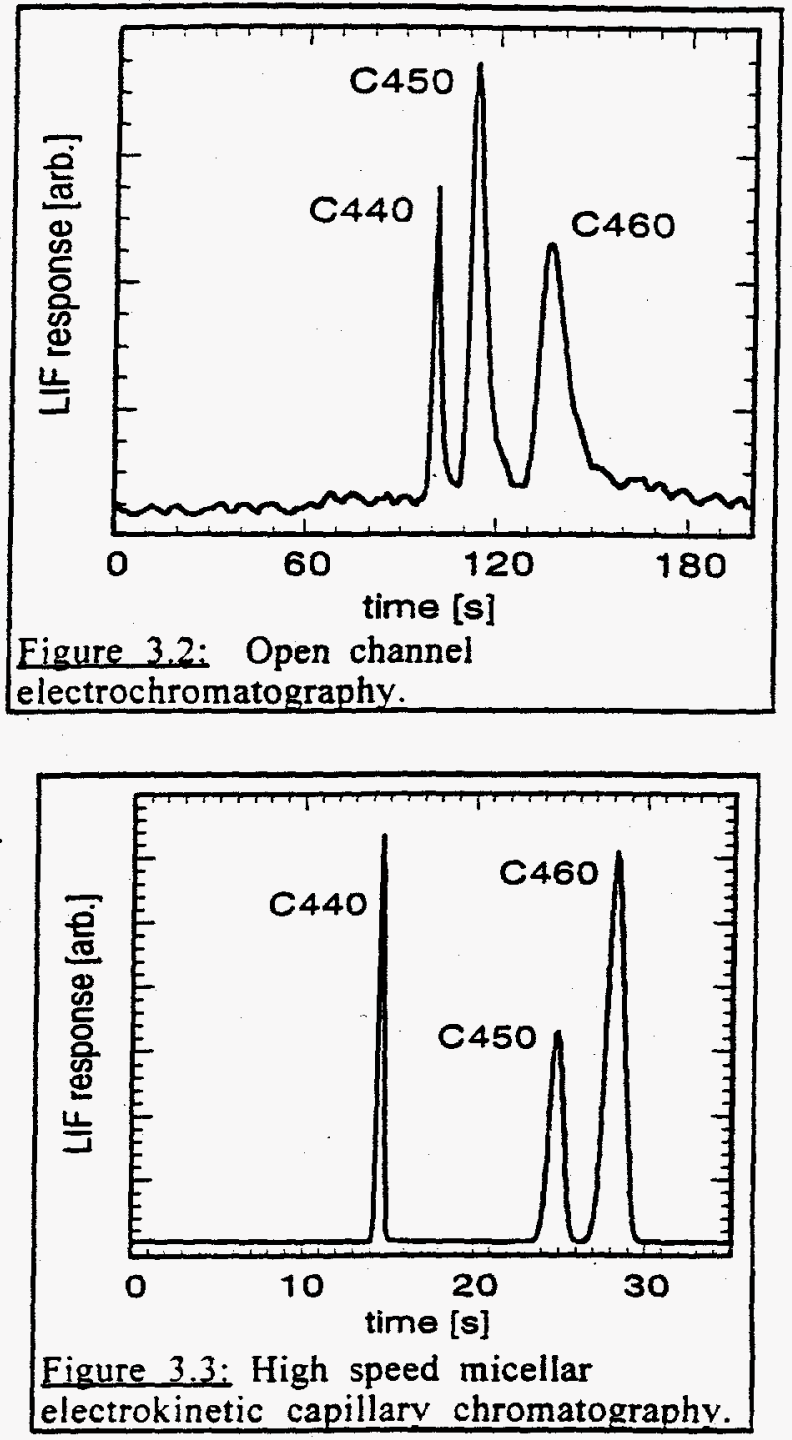
respectively. Improvements $\mathrm{i} n \mathrm{th}$ efficiency would be seen with better st a tionary phase coating, c h a n n e l geometry, and $s$ o $v$ e n t programming (shown below).

In Figure 3.3, fast M E C C separations are performed in 30 s generating

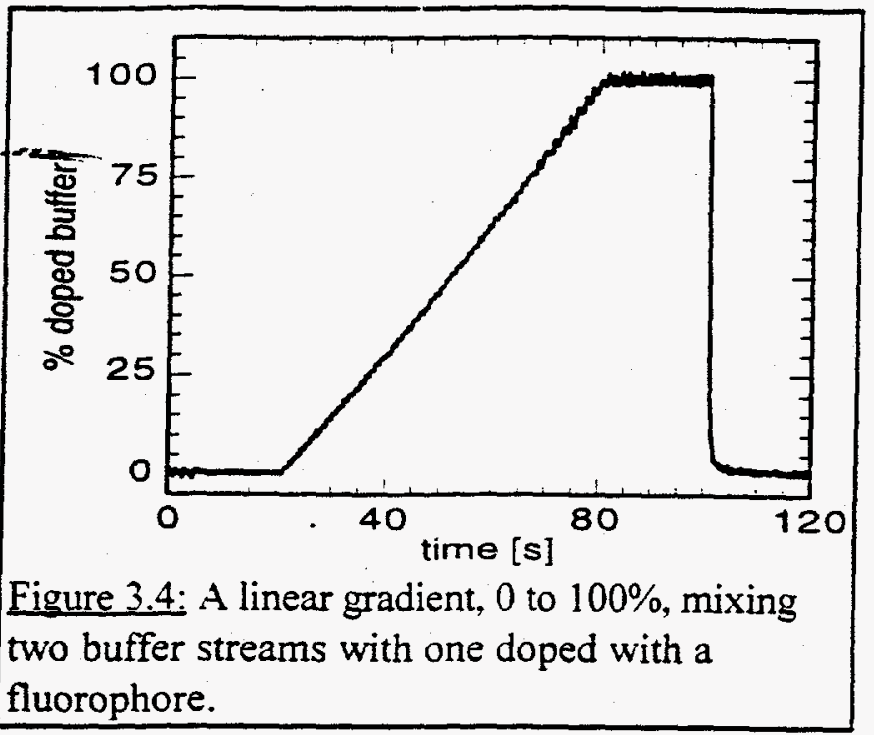
8800 and 3200

plates for the least and most retained components, respectively, using a cross microchip. Due to the high field strength, $500 \mathrm{~V} / \mathrm{cm}$, a loss in efficiency due primarily to mass transfer kinetics is observed between the unretained peak, C440, and the most retained peak, C460. Three primary advantages of MECC over open channel electrochromatography are a higher stationary phase density in the separation channel, the separation medium is replaceable, and fabrication is simpler because channel walls do not require coating with a stationary phase. The primary drawback is micelles elute from the column, and consequently, a finite separation window can be limiting for some separations.

Solvent programming can be performed on-chip by mixing two streams at a tee connector. Programmable, high voltage power supplies deliver precise electric potentials to the solvent reservoirs, and in turn, electrokinetic transport drives fluids and samples through the channel manifold. Mixing is rapid due to the small distances for diffusive homogenization of the solvents. Figure 3.4 displays a $60 \mathrm{~s}$ linear gradient of two buffer streams mixed at a tee intersection. One stream is doped with a fluorophore in order to demonstrate the quality and precision of the mixing. Gradients can be generated using step, linear, or nonlinear functions depending on the requirements of a given system. 


\section{Monolithically integrated microchip structures}

Many of the performance features of the microchip separation devices (small injection volumes with high reproducibility, millisecond separations) are due to the dexterity with which materials can be manipulated and the ability to make $c h$ a $n$ n e 1 interconnects with essentially zero dead volume. The fluid man ipulation capability has led to more exciting devices that monolithically integrate chemical reactions with $c h$ e $m$ i c al separations. As mentioned above, we have demonstrated microfabricated devices for both preand post-separation reactions. The precolum n reactor coupled $t 0$ a $n$ electrophoresis channel is the first published example of a monolithically in $t$ egrat ed c he m ic al microchip. As shown in Figure 4. 1 , two $r$ e a g e $n$ t reservoirs are connected by channels to a $r$ a c $t$ io $n$ chamber with a
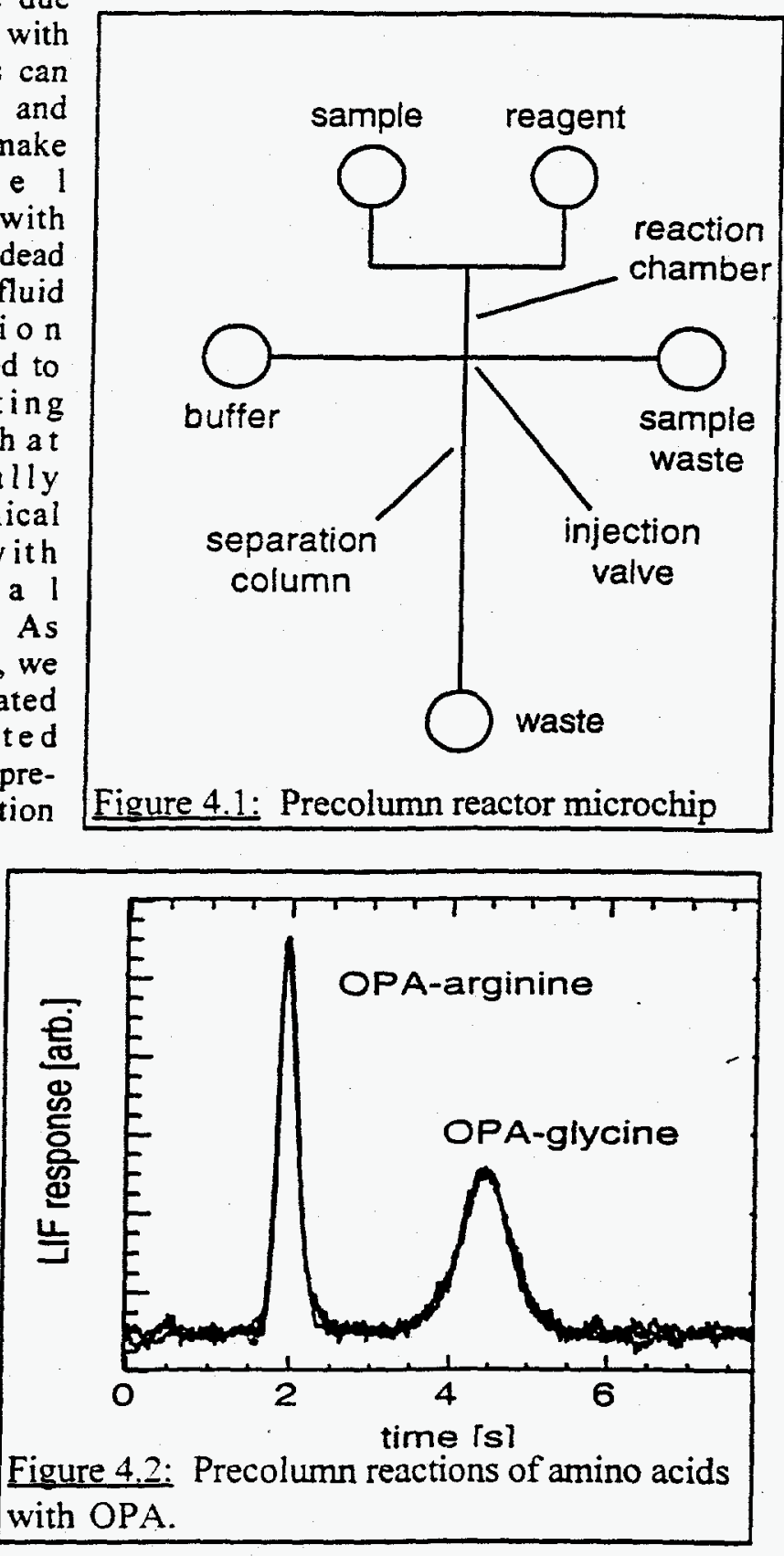


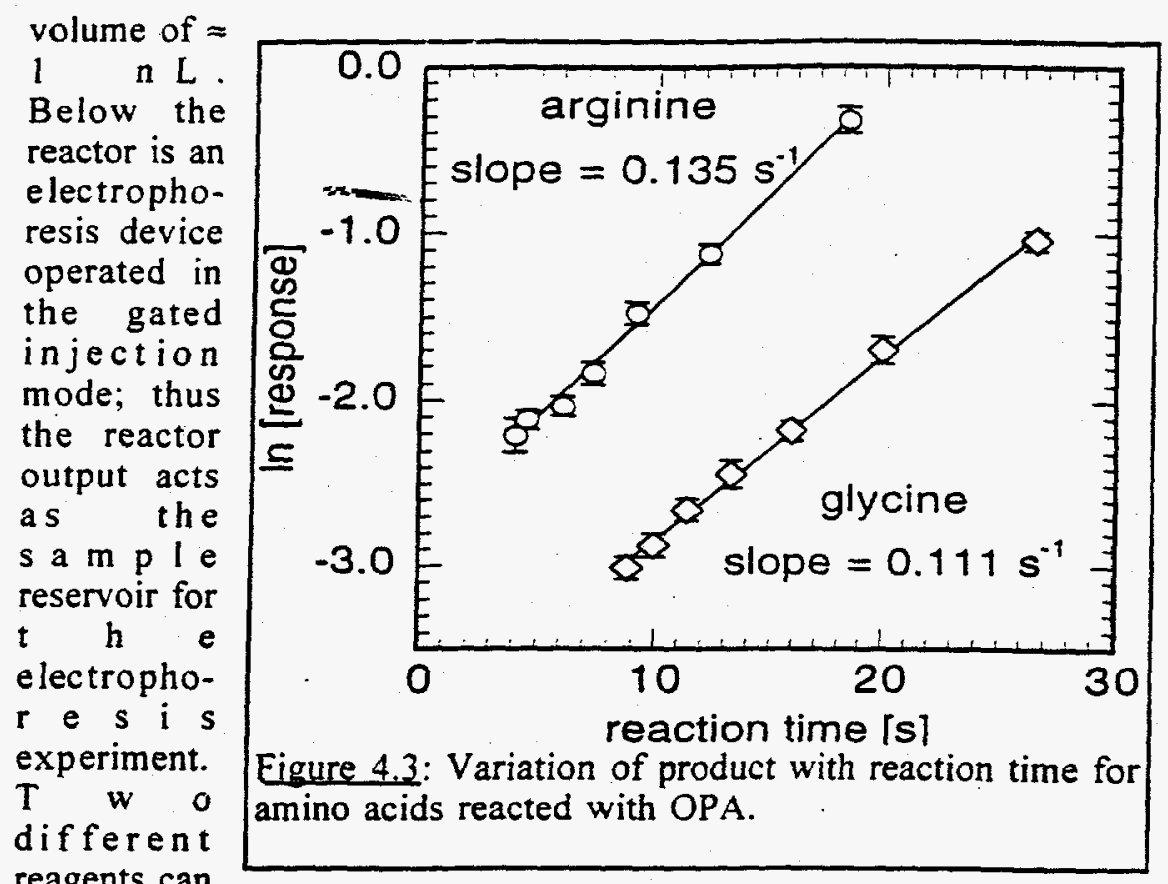
reagents can

be placed in separate reservoirs and electrokinetically pumped into the reaction chamber. The reaction time is determined by the velocity of the components through the reactor, which can be controlled by adjusting the applied potentials. The reaction products are normally directed to the sample waste reservoir but can be injected into the electrophoresis channel using the gated injection protocol described above. The device schematically shown in Figure 4.1 was first demonstrated using the reaction between amino acids and ophthaldialdehyde (OPA) to generate a fluorescent product [18]. Figure 4.2 shows three repetitive electropherograms for glycine and arginine reacted with OPA. The flow control is sufficiently precise to accurately determine the residence time of the reagents in the reactor. Therefore, reaction kinetics for parallel multiple reactions can be simultaneously determined by recording the area of each peak as a function of reaction time (Figure 4.3). The measured half-times of reaction were $5.1 \mathrm{~s}$ and $6.2 \mathrm{~s}$ for arginine and glycine, respectively. These half-times of reaction are comparable to the $4 \mathrm{~s}$ previously reported for alanine [22]. These reaction kinetics can be rapidly generated $(=5 \mathrm{~min}$ ) under computer control while consuming minuscule volumes of reagents $(\approx 100 \mathrm{~nL})$. This experiment demonstrates the potential advantages of performing chemical experiments from a computer keyboard!

More recently, we have demonstrated a monolithically integrated device similar to that of Figure 4.1 to perform DNA restriction fragment analysis [19], i.e., the two reagents were DNA (plasmid 
pBR322) and a restriction endonuclease (Hinf I). The channel walls were first coated with linear polyacrylamide to minimize electroosmotic flow and then pressure filled with $3 \%$ polyacrylamide in $50 \mathrm{mM}$ Tris-borate ( $\mathrm{pH} 8.2$ ), $5 \mathrm{mM}$ EDTA. The DNA and enzyme were electrophoretically loaded into a reactor with a volume of $700 \mathrm{pL}$ by applying a potential to the reagent reservoirs relative to the sample waste reservoir. The potential applied to the chip was temporarily removed to allow the reaction to proceed for 0.5 to $3 \mathrm{~min}$. Following the reaction, potentials were reapplied appropriately to inject the digested products onto the separation c olum $\mathrm{n}$ a $n \quad d$ electroph oretically separate them.

g a $t$ e c injection was used with an injection time of 10 s. The restriction fragments $w$ e $r$

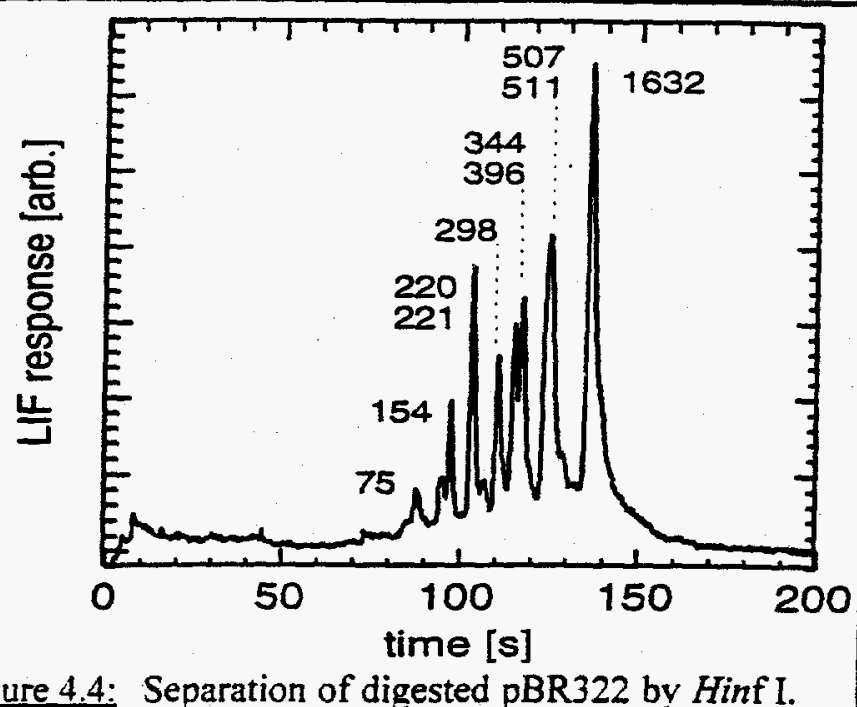

resolved in the presence of $1.0 \%(w / v)$ hydroxyethyl cellulose. Digested fragments were detected using laser induced fluorescence with a fluorescent intercalating dye, TOTO-1 (Molecular Probes, Inc.). The dye was placed only in the waste reservoir and electrophoretically pumped countercurrent to the separation. Figure 4.4 shows an electropherogram of the restriction fragments of the plasmid pBR322 digested with Hinf I enzyme for a reaction time of $2 \mathrm{~min}$. For this electropherogram the separation field strength was $375 \mathrm{~V} / \mathrm{cm}$ and the separation length was $65 \mathrm{~mm}$. The concentration of the undigested DNA was $125 \mathrm{ng} / \mu \mathrm{L}$ corresponding to $=30$ attomoles of material loaded into the reactor. More recently we have improved the resolving power of the fragment sizing as is shown in Figure 4.5. A DNA sample of pBR322 was digested with Hae III. The HEC was replaced with $3 \%(w / v)$ of linear polyacrylamide as a sieving media. The fragments were separated in a linear column of $25 \mathrm{~mm}$ at an electric field strength of $82 \mathrm{~V} / \mathrm{cm}$. Laser induced fluorescence was used for detection of the resolved fragments with an intercalating 
fluorescent dye (TO-PRO) to give detection limits of $\approx \operatorname{lng} / \mu \mathrm{L}$.

Figure 4.6 shows a simple cross type chip that was used to perform on-chip polymerase chain reaction (PCR) with integrated electrophoretic sizing. The indicated reservoir was used as the PCR reaction chamber boling a typical reaction volume of $10 \mu \mathrm{L}$. The other three reservoirs contained $1 \%$ HEC sieving buffer with the lower w a s t e reservoir additiona 1 l y containing TO$\mathrm{PRO}$ as a $\mathbf{n}$ in tercalat ing dye for fluores$c \mathrm{e} \in \mathrm{e}$ derection of DNA $f r a g$ ments. A small a mount $o$ $f$

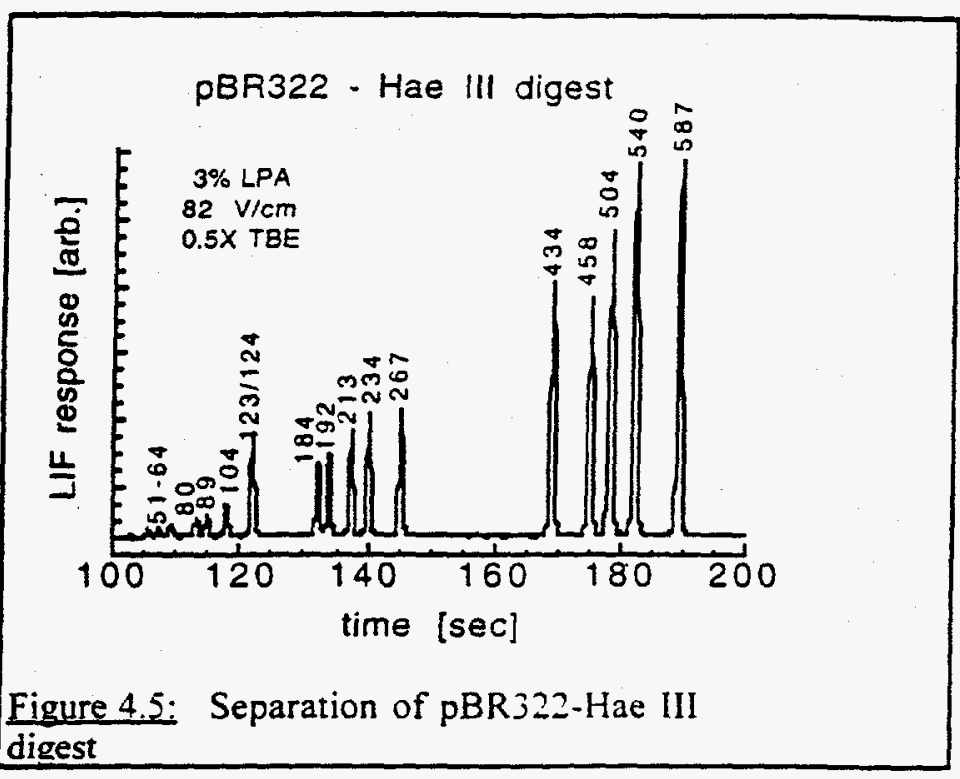
mineral

oil was placed on top of aqueous solutions in each $r$ e $s$ r voir. Thermal cycling was achieved by placing the entire chip on a Peltierbased temperature controller. After cycling, the chip was transported to a microchip elect roph ore sis station for analysis of the generated PCR products. The fragments

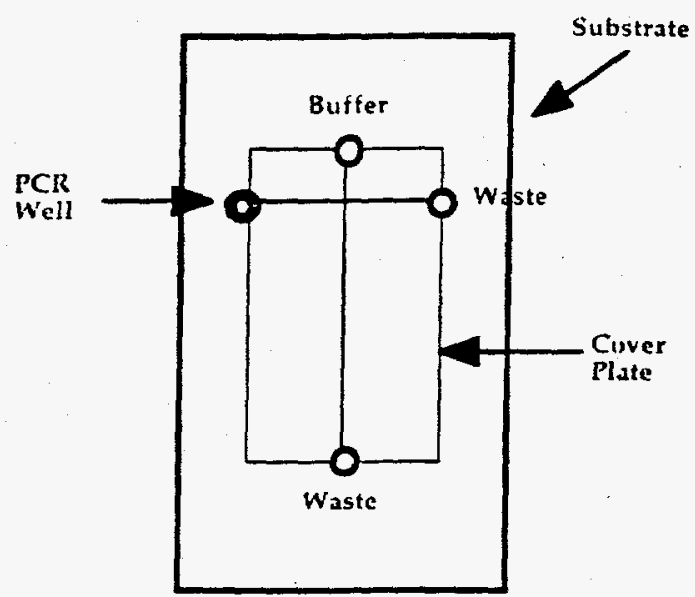

Figure 4.6 Microchip PCR/CE Device were detected as 
described above for the restriction digests. The results of an on-chip $P$ C R / C E analysis using AmpliT AQTM D $N \quad A$ polymerase (Perkin Elmer Cetus) to amplify a 500 bp sequence in lambda DNA template (25 cycles) are shown in Figure 4.7. The upper electropherogra $m$ shows the $500 \quad b$ p amplicon and a smaller primerdimer product. The size of the PCR product was confirmed by manually

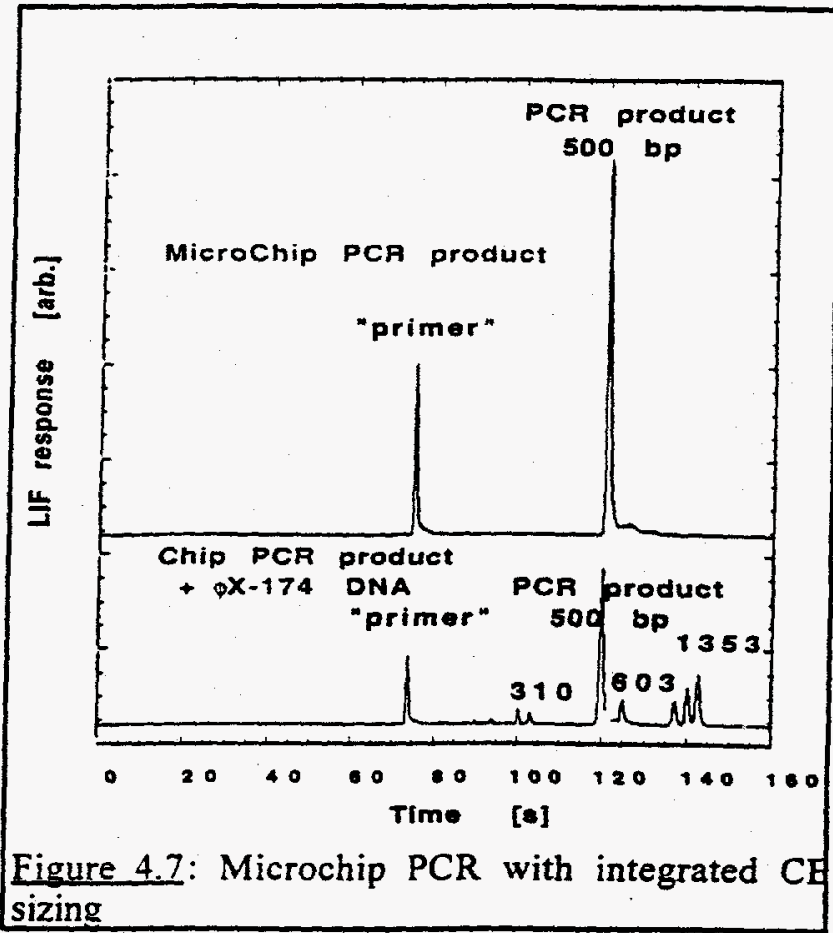
adding $F X-174$

(Hae III) sizing ladder to the reaction mixture and perfroming a second CE separation (bottom electropherogram). Both fragments produced by the PCR reaction have migrations times consistent with their expected sizes. The goal of this demonstration is to show that a very simple microchip device can include a PCR reactor, using conventionally sized sample volumes, and an integrated $C E$ sizing function. The simplicity and associated low cost of the device will allow the chip to be a disposable component. High throughput genetic diagnostic information could be obtained by multiplexing primer sets within a well and also by "horizontal" expansion to include multiple sample wells and CE separation channels.

The above results from our laboratory demonstrate state-of-the-art capabilities for chemical analysis in microchips incorporating both processing (chemical reactions) and separation (analysis) functionalities. While these devices are simplistic from a microfabrication perspective, they are exceedingly powerful from the standpoint of elucidating chemical information from minute quantities of materials. The monolithic integration of additional functional elements will provide ever greater chemical processing and informing power while maintaining fabrication simplicity. Monolithic integration will also allow economy of scale fabrication and the realization of low cost devices. 
16. van der Schoot, B. H., Jeanneraet, S., van den Berg, A., de Rooij, N. F., Anal. Methods Instrum., 1, 38 (1993).

17. Murakami, Y., Takeuchi, T., Yokayama, K., Tamiya, E., Darube, I., Suda, M., Anal. Chem., 65, 2731 (1993).

18. Jacobson, S.C., Hergenröder, R., Moore, A.W., Ramsey, J.M., Anal. Chem., 66, 4127 (1994).

19. Jacobson, S.C., Ramsey, J.M. Anal. Chem. 68, 720 (1996).

20. Jacobson, S.C., Koutny, L.B., Hergenröder, R., Moore, A.W, Ramsey, J.M. Anal. Chem., 66, 3472 (1994).

21. Adamson, A.W., "The Physical Chemistry of Surfaces", Ch. 4, Interscience Publishers, New York, (1960).

22. Butchner, E.C., Lowry, O.H. Anal. Biochem., 76, 502 (1976). 